\title{
Bitter melon (Momordica charantia) fruit extract ameliorates methotrexate-induced reproductive toxicity in male rats
}

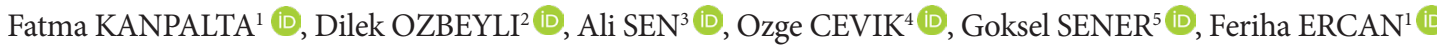 \\ ${ }^{1}$ Department of Histology and Embryology, School of Medicine, Marmara University, Istanbul, Turkey. \\ ${ }^{2}$ Pathology Laboratory Techniques, Vocational School of Health Sciences, Marmara University, Istanbul, Turkey. \\ ${ }^{3}$ Department of Pharmacognosy, School of Pharmacy, Marmara University, Istanbul, Turkey. \\ ${ }^{4}$ Department of Biochemistry, School of Medicine, Adnan Menderes University, Aydin, Turkey. \\ ${ }^{5}$ Vocational School of Health Service, Fenerbahce University, Istanbul, Turkey.
}

Corresponding Author: Feriha ERCAN

E-mail: eferiha@hotmail.com

Submitted: 23.02.2021 Accepted: 25.05.2021

\section{ABSTRACT}

Objective: Methotrexate (MTX) is a drug commonly used for the treatment of malign neoplastic and inflammatory diseases. Antioxidant and anti-inflammatory effects of bitter melon (BM) were reported. The aim of this study was to examine the effects of BM fruit extract on MTX-induced testicular and epididymal damage.

Materials and Methods: Sprague Dawley male rats were divided into three groups $(n=8)$ as control, MTX and MTX+BM. A single dose of MTX (20 mg/kg) was injected intraperitoneally to the MTX and MTX+BM groups. BM fruit extract (600 mg/kg) was applied to the MTX+BM group orally for 5 days. Testes were examined for general histopathology, proliferating and apoptotic cells. The epididymis samples were used for the evaluation of sperm morphology. Oxidative and inflammatory markers were analysed biochemically. Results: Increased abnormal spermatozoa, degenerated seminiferous tubules with increased apoptotic cells and decreased proliferative cells were observed in the MTX group. TNF- $\alpha$, IL-1 $\beta$, 8-hydroxy-2-deoxyguanosine and caspase-3 levels increased, superoxide dismutase and catalase levels decreased in both testis and epididymis samples. All these histological and biochemical parameters were ameliorated in the MTX+BM group.

Conclusion: Methotrexate causes testis damage by decreasing spermatogenic cells and increasing apoptosis through oxidative stress and inflammation. BM extract improves testis and epididymis damage with its possible anti-oxidant and anti-inflammatory effects.

Keywords: Methotrexate, Bitter melon, Testis, Epididymis, Oxidative stress

\section{INTRODUCTION}

Methotrexate (MTX), a folic acid anti-metabolite drug, is commonly used in the treatment of many clinical diseases such as romatoid arthritis, systemic lupus erythematosus, psoriasis and some malign neoplastic diseases [1]. Its use is limited due to its side effects including pansitopenia, infections, hepatotoxicity and neuropathy [2]. In addition, the male genital system is also adversely affected by MTX. An increase in degenerated seminiferous tubules with apoptotic cells and a decrease in proliferative cells in the germinal epithelium have been reported $[1,3-8]$. Various mechanisms have been proposed to explain its side effects, but not yet fully elucidated. MTX induces apoptosis by causing DNA damage $[1,3-5,7,8]$, disrupting the oxidant and anti-oxidant balance [3-9] and increasing inflammation [5, $6,10]$ thereby leading to toxicity in male reproductive systems.

Anti-oxidants and anti-inflammatory compounds such as folic and folinic acid [1], propolis [3], zinc [4], chrysin [5], protocateuchuic acid [6], vitamin B17 [7], apocynin [8] and agomelatine [10] have been indicated to prevent spermatogenic cells from MTX-induced male reproductive system damage. These studies have shown that anti-oxidants are thought to help protect testicular tissue against oxidative stress.

Momordica charantia, known as bitter melon (BM), is a vegetable belonging to Cucurbitaceae family. BM is grown in tropical and subtropical regions of many countries. This plant, rich in carbohydrates, proteins, fiber, minerals and vitamins, has been used for traditional treatment. Many parts of this plant (fruit, seed, root) are used in traditional treatments as well as being food. BM contains essential oils, flavonoids, phenolic acids, fatty acids, amino acids, lectins, etc. and they are responsible for its biological activity [11]. Many usefull effects of BM was reported such as anti-oxidant activity [12-14], anti-inflammatory effect $[15,16]$,

How to cite this article: Kanpalta E, Ozbeyli D, Sen A, Cevik O, Sener G, Ercan F. Bitter melon (Momordica charantia) fruit extract ameliorates methotrexate-induced reproductive toxicity in male rats. Marmara Med J 2021; 34: 222-228, doi: 10.5472/marumj.988941 
anti-diabetic property $[17,18]$, wound healing effect $[19,20]$, antiviral activity [21] and anticancer effect [22-26]. Protective effects of BM have been reported on damaged tissues such as liver [27], brain [12,16] and testis [17] in previous studies.

Yet, effects of BM against testis and epididymis damage induced by MTX have not been documented. So, the purpose of this study was to search the possible anti-oxidant and anti-inflammatory effects of BM fruit extract on testicular damage in MTX-induced rats.

\section{MATERIAL and METHODS}

\section{Experimental animals}

Twenty-four Sprague Dawley male rats (twelve weeks old) were purchased from the Marmara University Experimental Animals Research and Implementation Centre. They were transferred and kept in polycarbonate cages with soft rice husk bedding in a room controlled for ventilation (air exchange 18 time/hour), 12-h lightdark cycle, relative humidity $(50-60 \%)$ and temperature $\left(23-25^{\circ} \mathrm{C}\right)$ during the study. The animals were given balanced food and water ad libitum. This study was approved by Marmara University, School of Medicine, Animal Care and Use Committee (08.2020.mar).

\section{Preparation of the Bitter Melon Fruit Extracts}

Bitter melon fruits were collected from the rural area of Umurbey, Gemlik district of Bursa, Turkey in August 2019. The botanical determination of the plant was made by Dr. Ismail Senkardes. The voucher specimens have been stored in the Herbarium of School of Pharmacy, Marmara University (MARE No: 22446). BM fruit extract was prepared in the Marmara University Pharmacognosy Department. Briefly, fresh fruits with seeds were smashed by blender, and then, were macerated by ethanol 95\% (1000 mL) for $48 \mathrm{~h}$. The extraction process was repeated until the solvent becomes colourless. The filtrate was evaporated and concentrated at $40{ }^{\circ} \mathrm{C}$ using a rotary evaporator. The dried ethanol extract, obtained with a yield of $5.35 \%$, was stored at $+4{ }^{\circ} \mathrm{C}$ until the analysis.

\section{Experimental groups}

The rats were randomly divided into three groups $(\mathrm{n}=8)$ as control, MTX and MTX+BM. A single dose of MTX $(20 \mathrm{mg} / \mathrm{kg})$ was applied to MTX and MTX+BM groups by intaperitoneal injection. $\mathrm{MTX}+\mathrm{BM}$ group was given BM fruit extract dissolved in saline (600 $\mathrm{mg} / \mathrm{kg}$ ) by oral gavage for 5 days. BM fruit extract administration dose was decided according to a previous study of Subramani and Krishnamurthy (2019), that reported the therapeutic efficacy at a dose of $600 \mathrm{mg} / \mathrm{kg}$ [28]. The control and MTX groups were given saline for 5 days. The animals were decapitated and testis and epididymis samples were obtained at the end of the experiment.

\section{Measurement of superoxide dismutase, catalase, caspase-3, 8-hydroxy-deoxyguanosine, interleukin-1 $\beta$, and tumor necrosis factor $-\alpha$ levels}

The superoxide dismutase (SOD), catalase (CAT), caspase-3, 8-hydroxy-deoxyguanosine (8-OhDG), interleukin-1 $\beta$ (IL-1 $\beta$ ) and tumor necrosis factor (TNF)- $\alpha$ levels were measured in epididymis and testis homogenates by using commercial Enzyme Linked Immunosorbent Assay (ELISA) kits (Abbkine Inc. Wuhan, China). The results were given as ng/mg for CAT, 8 -OhDG and IL-1 $\beta$; U/mg for SOD; nmol pNA/mg for caspase-3 and pg/mg for TNF- $\alpha$ levels.

\section{Morphological evaluation of epididymal sperm}

The left caudal epididymis was cut into small pieces and a routine density gradient method was used to examine the sperm. For sedimentation, the supernatant was removed using 5 ml Earle's Balanced Salt Solution (Sigma, USA), then the pellet was diluted with sperm washing medium (SAGE, UK) and centrifuged. The pellet was then diluted with sperm preparation medium (SAGE, UK). Smears were stained with Diff-Quick kit (Medion Diagnostics, Grafelfing, Germany) for morphological evaluation. One hundred spermatozoa were evaluated under a photomicroscope at $400 \times$ magnification for tail, neck and head morphology [29].

\section{Light microscopic preparation}

The right testes were collected and fixed with 10\% neutral buffered formalin and processed for rouitine paraffin embedding technique. Paraffin sections $(5 \mu \mathrm{m})$ were stained with hematoxylin and eosin (H\&E) for general morphological evaluation, periodic acid Schiff (PAS) reaction for evaluation of basement membrane and evaluated under a photomicroscope (Olympus BX51, Tokyo, Japan). In each section, 20 seminiferous tubules were measured for germinal epithelium thickness and evaluated with histopathological Johnsen's score [30] at 200x magnification. Each tubule was scaled from 1 (absence of germinal epithelium) to 10 (complete spermatogenesis) according to the epithelial maturation.

\section{Proliferating cell nuclear antigen immunohistochemistry analysis}

Paraffin sections $(4 \mu \mathrm{m})$ were placed in a $57^{\circ} \mathrm{C}$ oven for 1 hour, then deparaffinized with xylene. After decreasing series of alcohol, they were put into $3 \%$ hydrogen peroxide solution. They were boiled in citrate buffer (10 mM; pH 6.0;20 min) for antigen retrivial. After washing in phosphate buffered saline (PBS), sections were incubated with blocking solution (SensiTek HRP Anti-Polyvalent Lab Pack, AEM080). Sections were incubated overnight $\left(4{ }^{\circ} \mathrm{C}\right)$ with rabbit anti-proliferation cell nuclear antigen (PCNA) primary polyclonal antibody (ab152112, Abcam, Cambridge, UK) in a humidified chamber. Slides were washed with PBS and incubated with biotinylated secondary antibody (20 min). After washing with PBS, the sections were placed in streptavidin peroxidase (20 min), washed with PBS, and applied 3, 3-diaminobenzidine tetrahydrochloride dihydrate (DAB) chromogen and counterstained with hematoxylin. In each section, PCNA-positive and negative cells were counted in 20 seminiferous tubules. The proliferation index was calculated by the percentage of PCNA-positive spermatogonia (PCNA-positive spermatogonia / total number of spermatogonia) in each seminiferous tubule. 


\section{Terminal deoxynucleotidyl transferase dUTP nick end labelling analysis}

The terminal deoxynucleotidyl transferase dUTP nick end labelling (TUNEL) method was used according to the instructions of kit (Apoptag Kit Plus Peroxidase, In Situ Apoptosis Detection Kit, Millipore, S7101, Temecula, CA, USA). Paraffin sections were counterstained with hematoxylin. In each section, TUNEL-positive cells were counted in 20 seminiferous tubules. Apoptotic index was calculated according to tubules which is containing three or more TUNEL-positive cells were reported as the percentage of twenty tubules.

\section{Statistical Analysis}

One-way ANOVA analysis of variance was used for data analysis and Tukey's multiple comparisons test was used for determination of differences between groups. The results were represented as mean \pm standard deviation (SD) using Graph Pad Prism Version 8.0 (Graph Pad Software, San Diego, USA). Significant difference was considered at the level of $\mathrm{p}<0.05$.

\section{RESULTS}

\section{Results of SOD, CAT, caspase-3, 8-OHdG, IL - $1 \beta$, and TNF - $\alpha$ levels}

SOD $(\mathrm{p}<0.001)$ and CAT $(\mathrm{p}<0.001)$ levels reduced, 8-OHdG $(\mathrm{p}<0.001)$, caspase-3 $(\mathrm{p}<0.01)$, TNF- $\alpha \quad(\mathrm{p}<0.001)$ and IL-1 $\beta$ $(\mathrm{p}<0.01)$ levels increased in epididymis samples of the MTX group compared to the control group. However, SOD $(\mathrm{p}<0.05)$ and CAT $(\mathrm{p}<0.05)$ levels were higher, $8-\mathrm{OHdG}(\mathrm{p}<0.01)$, caspase- 3 $(\mathrm{p}<0.01)$, TNF- $\alpha(\mathrm{p}<0.05)$ and IL-1 $\beta(\mathrm{p}<0.01)$ levels were lower in the MTX+BM group than the MTX group (Figure 1).
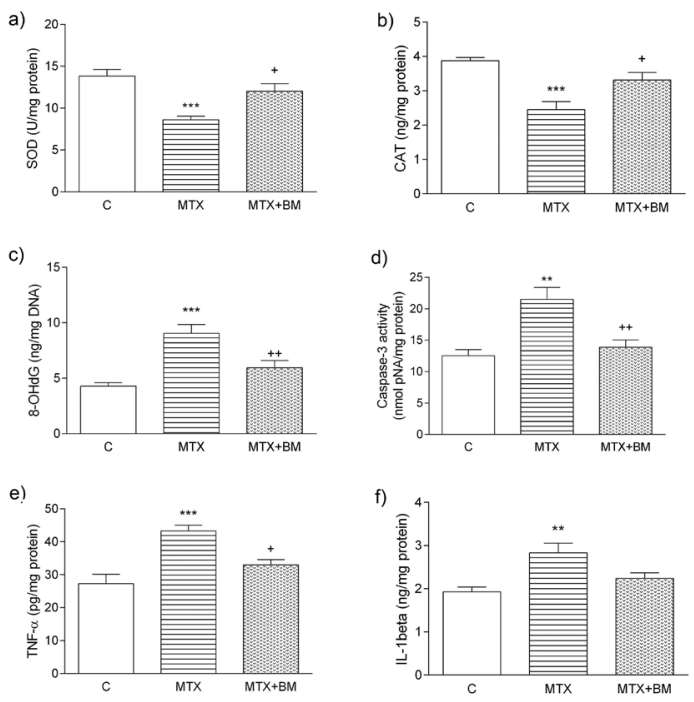

Figure 1. SOD (a), CAT (b), 8-OHdG (c), caspase-3 (d), TNF- $\alpha$ (e) and $I L-1 b(f)$ levels of epididymal tissues are seen in the experimental groups. ${ }^{* *} p<0.01$ and ${ }^{* * *} p<0.001$ compared to control (C) group, + $p<0.05$ and $++p<0.01$ compared to $M T X+B M$ group.
SOD $(\mathrm{p}<0.001)$ and CAT $(\mathrm{p}<0.01)$ levels decreased, 8-OHdG ( $<<0.001)$, caspase-3 ( $<<0.001)$, TNF- $\alpha(\mathrm{p}<0.001)$ and IL-1 $\beta(p<0.01)$ levels increased in the testis of the MTX group compared to the control group. But, SOD $(\mathrm{p}<0.05)$ and CAT $(\mathrm{p}<0.05)$ levels increased, 8-OHdG $(\mathrm{p}<0.01)$, caspase-3 $(\mathrm{p}<0.01)$, TNF- $\alpha(\mathrm{p}<0.01)$ and IL-1 $\beta(\mathrm{p}<0.01)$ levels decreased in the MTX+BM group compared to the MTX group (Figure 2).
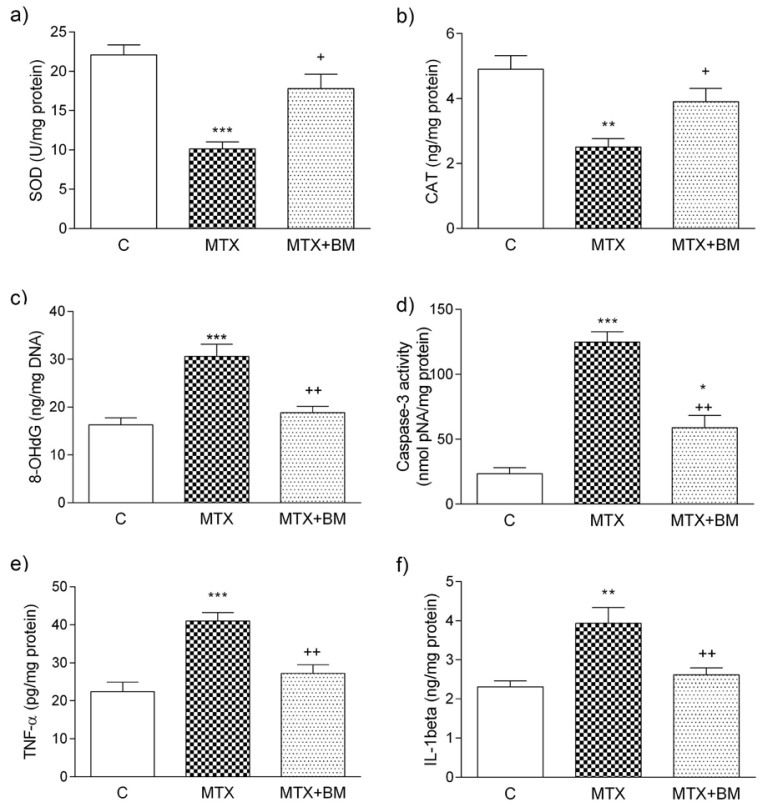

Figure 2 SOD (a), CAT (b), 8-OHdG (c), caspase-3 (d), TNF- $\alpha(e)$ and $I L-1 b(f)$ levels of testis tissues are seen in the experimental groups. ${ }^{*} p<0.05,{ }^{* *} p<0.01,{ }^{* *} p<0.001$ compared to control (C) group. $+p<0.05$, $++p<0.01$ compared to MTX group.

\section{Histopathological results}

According to our Diff Quick stained epididymal sperm morphological analysis, normal and abnormal sperms with tail, neck and head defects were seen in each of the experimental groups (Figure $3 \mathrm{~A}_{1}-\mathrm{C}_{1}$ ). Spermatozoa with head $(\mathrm{p}<0.001)$, neck $(\mathrm{p}<0.05)$ and tail $(\mathrm{p}<0.05)$ abnormalities increased and normal spermaotozoa decreased $(\mathrm{p}<0.001)$ in the MTX group compared to the control group. However, spermatozoa with head $(\mathrm{p}<0.01)$ and neck abnormalities decreased and spermatozoa with tail abnormalities $(\mathrm{p}<0.05)$ increased in the MTX+BM group compared to the MTX group (Figure $3 \mathrm{D}_{1}$ ) 


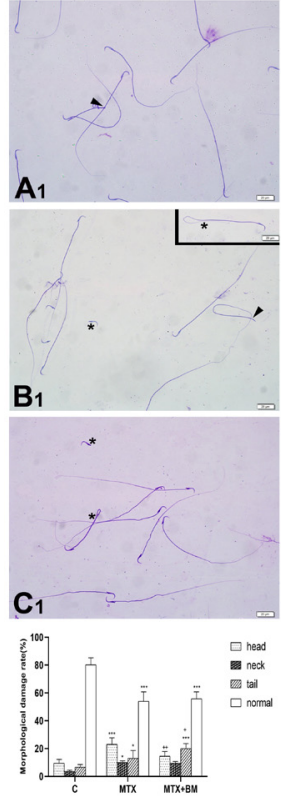

$\mathbf{D}_{1}$
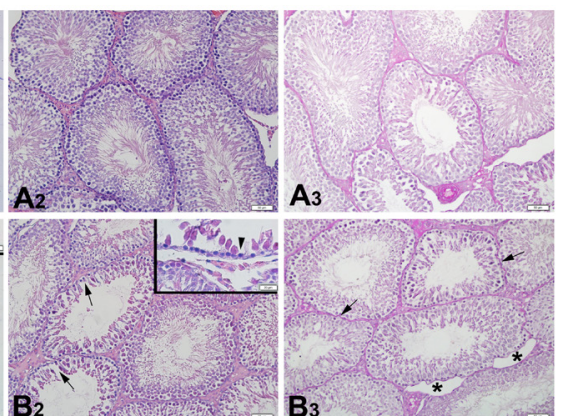

$\mathrm{B}_{2}$
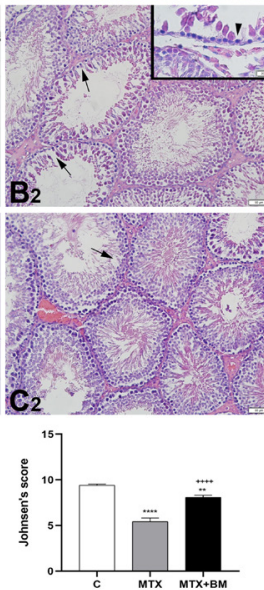

$\mathbf{D}_{2}$
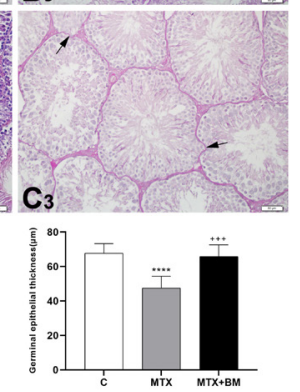

$\mathrm{D}_{3}$
Figure 3. Representative light micrographs of spermatozoa $\left(A_{-}\right.$ $\left.C_{1}\right)$, testis $\left(A_{2}-C_{3}\right)$, sperm morphologic damage ratio $\left(D_{1}\right)$, germinal epithelium thickness $\left(D_{2}\right)$ and Johnsen's histopathological scores $\left(D_{3}\right)$ are seen in the experimental groups. Normal spermatozoa and spermatozoa with head (arrow head) and tail $\left(^{*}\right)$ anomalies are seen in the control $\left(A_{1}\right), M T X\left(B_{1}\right)$ and $M T X+B M\left(C_{1}\right)$ groups. Normal seminiferous tubules with germinal epithelium $\left(A_{2}\right)$ and regular PAS positive basement membrane $\left(A_{3}\right)$ are seen in the control group. Degenerated seminiferous tubules with dilatation of the germinal epithelial cells (arrow), decreased number of germinal epithelial cells (arrowhead) are seen in MTX group $\left(B_{2}\right)$. PAS positive stained irregular basement membrane (arrow) and large vacuol formation in between the seminiferous tubules (asterisk) are seen in this group $\left(B_{3}\right)$. Normal seminiferous tubules and some degenerated tubules with dilatation of germinal epithelium (arrow) are seen in $M T X+B M$ group. PAS positive irregular basement membrane in some area (arrow) are seen in this group $\left(C_{3}\right) . A_{1}-C_{1}$ : Diff-Quick staining. Scale bar: $20 \mu \mathrm{m} . A_{2}-C_{2}: H \& E$ staining. $A_{3}-C_{3}$ : $P A S$ staining. Scale bar: 50 $\mu \mathrm{m}$, inset $B_{2}: 20 \mu \mathrm{m} .{ }^{*} p<0.05,{ }^{* *} p<0.01,{ }^{* * *} p<0.001$ and ${ }^{* * * *} p<0.0001$ compared to control (C) group. $+p<0.05,++p<0.01,+++p<0.001$ and $++++p<0.0001$ compared to MTX group.

Regular seminiferous tubules with basement membrane, spermatogenic and Sertoli cells and sperms in lumen were seen in the control group (Figure $3 \mathrm{~A}_{2}, \mathrm{~A}_{3}$ ). Degenerated seminiferous tubules with dilatations of the germinal epithelium, reduction of the germinal cell line, irregular basement membrane and large dilatations between the seminiferous tubules were observed in the MTX group (Figure $3 \mathrm{~B}_{2}, \mathrm{~B}_{3}$ ). Although, some dilatations were found in seminiferous tubules, quite normal appearing seminiferous tubules were observed in the $\mathrm{MTX}+\mathrm{BM}$ group (Figure $\left.3 \mathrm{C}_{2}, \mathrm{C}_{3}\right)$. Johnsen's score $(\mathrm{p}<0.001)$ and germinal epithelium thickness $(\mathrm{p}<0.001)$ decreased in the MTX group compared to the control group. However, Johnsen's score $(\mathrm{p}<0.001)$ and germinal epithelium thickness $(\mathrm{p}<0.0001)$ increased in the $\mathrm{MTX}+\mathrm{BM}$ group compared to the MTX group (Figure $3 \mathrm{D}_{2}, \mathrm{D}_{3}$ ).

\section{PCNA immunohistochemistry results}

PCNA-positive cells were observed as dark brown colour in seminiferous tubules of the experimental groups (Figure $4 \mathrm{~A}_{1}$ $\left.\mathrm{C}_{1}\right)$. Proliferation index was lower $(\mathrm{p}<0.0001)$ in the MTX and $\mathrm{MTX}+\mathrm{BM}$ groups compared to the control group (Figure $4 \mathrm{D}_{1}$ ).

\section{TUNEL results}

TUNEL-positive cells were seen as dark brown colour in all of the experimental groups (Figure $4 \mathrm{~A}_{2}-\mathrm{C}_{2}$ ). However, the number of TUNEL positive cells was the highest in the MTX group (Figure $\left.4 \mathrm{~B}_{2}\right)$. Apoptotic index was higher in the MTX group $(\mathrm{p}<0.0001)$ compared to the control group and this apoptotic index value was lower in the MTX+BM group $(\mathrm{p}<0.0001)$ compared to the MTX group (Figure $4 \mathrm{D}_{2}$ ).

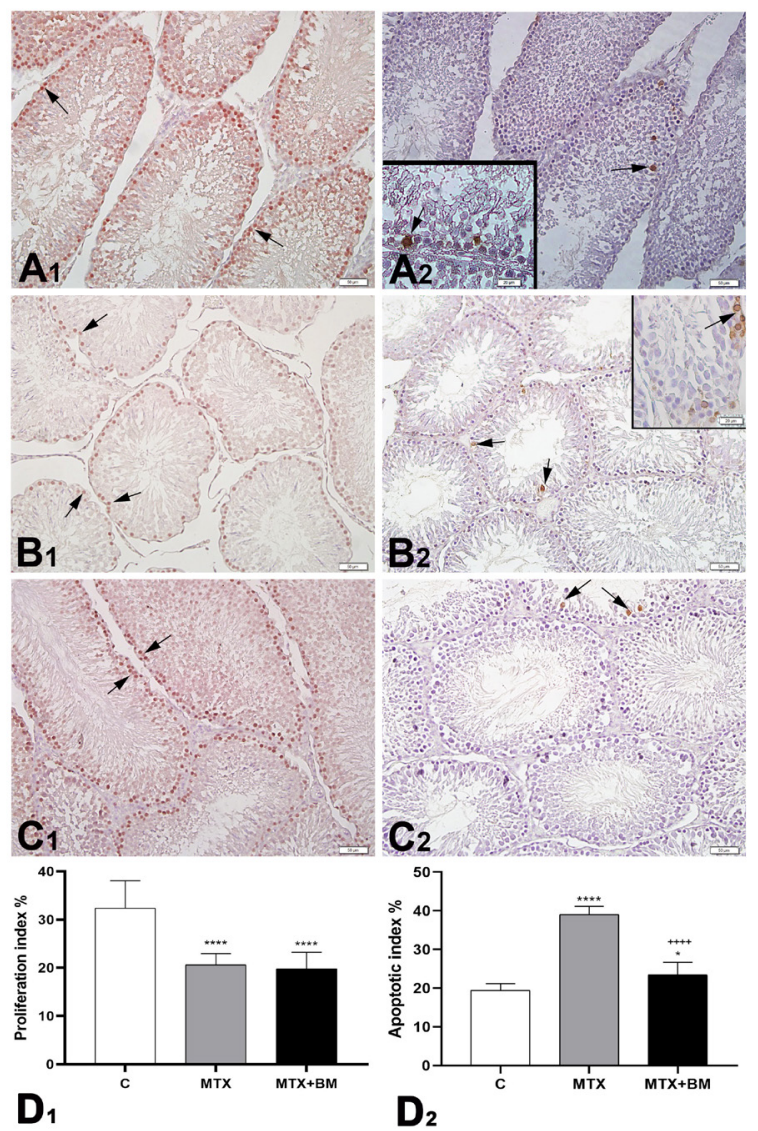

Figure 4. Representative light micrographs of PCNA-immunostained $\left(A_{1}-C_{1}\right)$ and TUNEL-stained $\left(A_{2}-C_{2}\right)$ testis samples, proliferation index $\left(D_{1}\right)$ and apoptotic index $\left(D_{2}\right)$ are seen in the experimental groups. Normal seminiferous tubules with numerous PCNA-positive (arrow, $A_{1}$ ), a few number of TUNEL-positive (arrow, $A_{2}$ ) spermatogenic cells are seen in the control group. A few number of PCNA-positive (arrow, $B_{1}$ ), increased number of TUNEL-positive (arrow, $B_{2}$ ) spermatogenic cell are seen in the MTX group. Normal seminiferous tubules with many PCNApositive cells (arrow, $C_{1}$ ), but decrease number of TUNEL-positive (arrow, $C_{2}$ ) spermatogenic cells are seen in the $M T X+B M$ group. Scale bar: 50 $\mu \mathrm{m}$; insets in $A_{2}$ and $B_{2}: 20 \mu \mathrm{m} .{ }^{*} p<0.05$ and ${ }^{* * * *} p<0.0001$ compared to control group, $++++p<0.0001$ compared to MTX group. 


\section{DISCUSSION}

In the present study, the ameliorating effects of BM on MTX induced testicular and epididymal damage were demonstrated by histological, immunohistochemical and biochemical methods. The results of this study showed that MTX induction caused changes in testicular morphology with decreasing proliferative cells and increasing apoptotic cells, increase of abnormal spermatozoa, testicular and epididymal oxidative stress and inflammation. BM fruit extract treatment ameliorated these histopathological and biochemical alterations in MTXinduced testis and epididymis damage by its anti-oxidant and anti-inflammatory properties.

Chemotherapeutic agents are used for treatment but they might cause excessive reactive oxigen species (ROS) accumulation. ROS increase causes infertility by disrupting the process of the male genital system. Therefore, anti-oxidant compounds are needed to protect the body from oxidative stress damage [29]. It was shown that the oxygen radical level of testis, epididymis and semen fluid are important and critical to preserve sperm vitality and function. Increase of ROS level in these tissues causes DNA damage or lipid peroxidation in spermatozoa; thus sperm capacitation, maturation and even vitality are negatively affected and fertility is reduced [31]. MTX is also a chemotherapeutic agent which is used in monotherapy or combined with other medicines for treatments of inflammatory and some malign neoplastic diseases $[1,2]$. A decrease in the activities of antioxidant endogenous enzymes SOD and CAT has been shown in MTX-induced testicular damage [2, 5, 7, 11]. Benefical effects of BM such as anti-oxidant [12, 14], antiinflammatory $[15,16]$ and anti-apoptotic activity, have been reported [17]. It was observed that BM aqueous extract reduced mitochondrial ROS in mice with restriction stress and prevented liver damage through its anti-inflammatory effect by reducing inducible nitric oxide synthase and the most protective effect of BM extact was found in $750 \mathrm{mg} / \mathrm{kg}$ dose [27]. In an another study, oral administration of BM fruit ethanolic extract in 250 and $500 \mathrm{mg} / \mathrm{kg}$ doses to diabetes induced testis damage showed anti-oxidant and anti-apoptotic effects, but BM extract was more protective in high doses [17]. In parallel with previous studies, it was found that oral administration of BM extract at a dose of $600 \mathrm{mg} / \mathrm{kg}$ has been found to have anti-oxidant, anti-apoptotic and anti-inflammatory effects in MTX-induced testicular and epididymis damage.

Methotrexate treatment increases ROS and lowers anti-oxidant defense, which results in increase in inflammation-related markers. Persistent inflammation has been shown to produce more ROS, which worsens oxidative damage [1, 2, 4-8]. TNF- $\alpha$ and IL- $1 \beta$ are cytokines that cause inflammatory reaction and control the inflammatory process. Some studies, have shown that MTX increases the levels of TNF- $\alpha$ and IL-1 $\beta$ in MTXinduced testis and epididymis damage in rats $[7,8]$. BM has been shown to prevent depressive-like behaviors in mice by reducing proinflammatory cytokines such as TNF- $\alpha$ and IL-1 $\beta$ levels in hippocampus [19]. In another study, BM was shown to reduce mitochondrial ROS in mice with restriction stress and prevented liver damage through its anti-inflammatory effect by reducing inducible nitric oxide synthase [30]. In this study, TNF- $\alpha$ and IL- $1 \beta$ levels rised in both testis and epididymis samples in MTX group, so our study showed that MTX-induced damage in those tissues was associated with inflammation. BM treatment decreased these inflammatory markers to the control levels in both testis and epididymis samples.

8 -OHdG which is formed by ROS in damaged tissue, is a marker of DNA damage. Previous studies have shown that MTX treatment increases $8-\mathrm{OHdG}$ in testis tissue [5]. Caspase-3 is the main executor caspase in the apoptotic pathway, its increase suggests apoptosis activation. A study has been shown to increase caspase-3 activity in the testis of animals treated with MTX [8]. Moreover, it has been shown that there is an increase in the number of apoptotic cells in the testis in experimental animals administered MTX $[1,4,5]$. In diabetic rats, BM has been shown to increase testicular anti-oxidant enzymes, reduce caspase-3 activity and inhibit apoptosis, resulting in histopathological improvement [20]. BM has been shown to inhibit the activation of JNK3/c-Jun/Fas-L and JNK3/cytochrome C/caspase-3 signaling cascades and prevent apoptosis due to its anti-oxidant properties during cerebral ischemia/reperfusion injury [15]. In an in vitro study, it has been shown that MTX causes apoptosis and autophagy in spermatocyte cell line via formation of ROS [10]. Parallel to these findings, an increase in 8-OHdG level and caspase- 3 activity in the epididymis and testis samples and an increase in apoptotic cells in the testis in MTX group has been observed in our study. However, BM treatment in MTX applied rats reduced caspase- 3 activity and $8-\mathrm{OHdG}$ level in both the testis and epididymis samples and apoptotic index in the testis samples.

It was reported that chemotherapeutic agents cause male genital system toxicity such as damage to the seminiferous tubules of the testis and epididymis, reducing sperm count and leading to genetic mutations in sperms [1,3-5]. It has been shown in many studies that these harmful effects are caused by oxidant damage $[3-6,8-10]$, inflammation $[5,6,10]$, DNA damage and apoptosis [1, 3-7] and decreased FSH, LH and testosteron hormone levels in the serum $[4,7,8,10,17]$. Previous studies have shown that MTX causes a decrease in proliferation $[3,4]$, an increase in apoptosis and DNA damage in the testis, also it decreases the number of spermatozoa and increases the number of abnormal spermatozoa [1, 3-5]. Parallel to the previous studies, we have observed decrease in endogeneous anti-oxidant SOD and CAT activities, as well as an increase in caspase-3 activity and 8-OHdG level in both the testis and epididymis samples in the MTX group. Histological findings showed that increased degenerated seminiferous tubules with a decrease in proliferative cells and an increase in apoptotic cells and increased abnormal spermatozoa were present in the MTX group. BM extract amelioriated MTX-induced testicular and epididymal damage via increase of endogenous anti-oxidant level and inhibition of apoptotic activity.

As a result, MTX is used for the treatment of many diseases, but it has side effects on male genital system toxicity. MTX leads to a decrease in anti-oxidant defence, an increase in inflammation and DNA damage in both testis and epididymis. Moreover, MTX 
causes damage in the seminiferous tubules of the testes with a decrease in the germinal epithelium thickness and proliferation. It also increases apoptosis and abnormal spermatozoa. Our study showed that aqueous fruit extract of BM protects MTXinduced testicular and epididymal damage in rats by its antioxidant, anti-apoptotic and anti-inflammatory response. The use of aqueous fruit extract of bitter melon may be an effective way to ameliorate potential male fertility in the MTX-induced damage. The limitation of this study is a need for further studies with different dose and time adjustments to obtain more accurate results.

\section{Acknowledgements}

The authors would like to thank botanist Ismail Senkardes, PhD, (Department of Pharmaceutical Botany, Faculty of Pharmacy, Marmara University, Istanbul, Turkey) for his help for identification of the plant material.

\section{Compliance with Ethical Standards}

Ethical Approval: This study was approved by Marmara University, School of Medicine, Animal Care and Use Committee (08.2020. mar).

Financial Support: The authors have no relevant financial information to disclose.

Conflict of Interest: The authors declare that there are no conflicts of interest.

Author Contributions: F.K., D.O., G.S. and F.E. contributed to the conception and design. F.K., D.O., A.S., O.C., G.S. and F.E. performed experiments and did data collection, F.K., G.S. and F.E. analyzed data. F.K., G.S. and F.E. contributed to the writing and F.E. did the critical revision of the article. All authors approved the final version of the article.

\section{REFERENCES}

[1] Padmanabhan S, Tripathi D, Vikram A, Ramarao P, Jena GB. Methotrexate-induced cytotoxicity and genotoxicity in germ cells of mice: intervention of folic and folinic acid. Mutat Res 2009; 673:43-52. doi: 10.1016/j.mrgentox.2008.11.011

[2] Romao VC, Lima A, Bernardes M, Canhao H, Fonseca JE. Three decades of low-dose methotrexate in rheumatoid arthritis: can we predict toxicity? Immunol Res 2014; 60:289310. doi: 10.1007/s12026.014.8564-6

[3] Sonmez MF, Cilenk KT, Karabulut D, et al. Protective effects of propolis on methotrexate-induced testis injury in rat. Biomed Pharmacother 2016; 79:44-51. doi: 10.1016/j. biopha.2016.02.002.

[4] Maremanda KP, Jena GJB. Methotrexate-induced germ cell toxicity and the important role of zinc and SOD1: Investigation of molecular mechanisms. Biochem Biophys Res Commun 2017; 483:596-601. doi: 10.1016/j.bbrc.2016.12.098.

[5] Belhan S, Comakli S, Kucukler S, Gulyuz F, Yildirim S, Yener Z. Effect of chrysin on methotrexate-induced testicular damage in rats. Andrologia 2019; 51: e13145. doi: 10.1111/and.13145
[6] Owumi SE, Ochaoga SE, Odunola OA, Farombi EO. Protocatechuic acid inhibits testicular and epididymal toxicity associated with methotrexate in rats. Andrologia 2019; 51(9):e13350. doi: 10.1111/and.13350

[7] Felemban SG, Aldubayan MA, Alhowail AH, Almami IS. Vitamin B17 ameliorates methotrexate-induced reproductive toxicity, oxidative stress, and testicular injury in male rats. Oxid Med Cell Longev 2020; 2020:4372719. doi: 10.1155/2020/4372719

[8] Sarihan K, Yardimoglu M, Eraldemir F, Yazir Y, Acar E. Protective effects of apocynin on damaged testes of rats exposed to methotrexate. Turk J Med Sci 2020; 50:1409-20. doi: 10.3906/sag-1909-52

[9] Xiong S, Song D, Xiang Y, et al. Reactive oxygen species, not $\mathrm{Ca}(2+)$, mediates methotrexate-induced autophagy and apoptosis in spermatocyte cell line. Basic and Clin Pharmacol Toxicol 2020; 126:144-52. doi: 10.1111/bcpt.13306

[10] Aslankoc R, Ozmen O, Ellidag HY. Ameliorating effects of agomelatine on testicular and epididymal damage induced by methotrexate in rats. J Biochem Mol Toxicol 2020; 34:e22445. doi: 10.1002/jbt.22445

[11] Sur S, Ray RB. Bitter Melon (Momordica Charantia), a nutraceutical approach for cancer prevention and therapy. Cancers 2020; 12:2064. doi: 10.3390/cancers12082064

[12] Gong J, Sun F, Li Y, et al. Momordica charantia polysaccharides could protect against cerebral ischemia/reperfusion injury through inhibiting oxidative stress mediated c-Jun N-terminal kinase 3 signaling pathway. Neuropharmacology 2015; 91 :12334. doi: 10.1016/j.neuropharm.2014.11.020

[13] Aljohi A, Matou-Nasri S, Ahmed N. Antiglycation and Antioxidant Properties of Momordica charantia. PLOS One. 2016; 11:e0159985-e. doi: 10.1371/journal.pone.0159985

[14] Chen F, Huang G, Yang Z, Hou Y. Antioxidant activity of momordica charantia polysaccharide and its derivatives. Int J Biol Macromol 2019; 138:673-80. doi: 10.1016/j. ijbiomac.2019.07.129

[15] Solomon U, Ashorobi R. Evaluation of anti-inflammatory and membrane stabilizing property of aqueous leaf extract of Momordica charantia in rats. Afr J Biomed Res 2009; 9:11924. doi: 10.4314/ajbr.v9i2.48892

[16] Deng Z, Yuan C, Yang J, et al. Behavioral defects induced by chronic social defeat stress are protected by Momordica charantia polysaccharides via attenuation of JNK3/PI3K/AKT neuroinflammatory pathway. Ann Transl Med 2019; 7(1):6. doi: 10.21037/atm.2018.12.08

[17] Soliman GA, Abdel-Rahman RF, Ogaly HA, et al. Momordica charantia Extract Protects against Diabetes-Related Spermatogenic Dysfunction in Male Rats: Molecular and Biochemical Study. Molecules 2020; 25(22): 5255. doi: $10.3390 /$ molecules 25225255

[18] Kim SK, Jung J, Jung JH, et al. Hypoglycemic efficacy and safety of Momordica charantia (bitter melon) in patients with type 2 diabetes mellitus. Complement Ther Med. 2020; 52:102524. doi: 10.1016/j.ctim.2020 
[19] Piskin A, Altunkaynak BZ, Tumentemur G, Kaplan S, Yazici OB, Hokelek M. The beneficial effects of Momordica charantia (bitter gourd) on wound healing of rabbit skin. J Dermatolog Treat 2014; 25:350-7. doi: 10.3109/09546.634.2012.713459

[20] Singh R, Garcia-Gomez I, Gudehithlu KP, Singh AK. Bitter melon extract promotes granulation tissue growth and angiogenesis in the diabetic wound. Adv Skin Wound Care 2017; 30:16-26. doi: 10.1097/01.ASW.000.050.4758.86737.76

[21] Schreiber CA, Wan L, Sun Y, Lu L, Krey LC, Lee-Huang S. The antiviral agents, MAP30 and GAP31, are not toxic to human spermatozoa and may be useful in preventing the sexual transmission of human immunodeficiency virus type 1. Fertil Steril 1999; 72:686-90. doi: 10.1016/s0015-0282(99)00302-7

[22] Pitchakarn P, Ogawa K, Suzuki S, et al. Momordica charantia leaf extract suppresses rat prostate cancer progression in vitro and in vivo. Cancer Sci 2010; 101:2234-40. doi: 10.1111/ j.1349-7006.2010.01669

[23] Claflin AJ, Vesely DL, Hudson JL, et al. Inhibition of growth and guanylate cyclase activity of an undifferentiated prostate adenocarcinoma by an extract of the balsam pear (Momordica charantia abbreviata). Proc Natl Acad Sci U S A 1978;75:98993. doi: 10.1073/pnas.75.2.989

[24] Sumanth M, Chowdary GN. Antimutagenic activity of aqueous extract of Momordica charantia. Int J Biotech Mol Biol 2010; 1:42-6. doi: 10.5897/IJBMBR.9000003

[25] Kaur M, Deep G, Jain AK, et al. Bitter melon juice activates cellular energy sensor AMP-activated protein kinase causing apoptotic death of human pancreatic carcinoma cells. Carcinogenesis 2013; 34:1585-92. doi: 10.1093/carcin/bgt081

[26] Fan X, He L, Meng Y, Li G, Li L, Meng Y. a-MMC and MAP30, two ribosome-inactivating proteins extracted from Momordica charantia, induce cell cycle arrest and apoptosis in A549 human lung carcinoma cells. Mol Med Rep 2015; 11:3553-8. doi: 10.3892/mmr.2015.3176.

[27] Deng Y, Tang Q, Zhang Y, et al. Protective effect of Momordica charantia water extract against liver injury in restraint-stressed mice and the underlying mechanism. Food Nutr Res 2017; 61:1348864. doi: 10.1080/16546.628.2017.1348864

[28] Subramani B, Krishnamurthy B. Effects of Momordica Charantia (Bitter gourd) on oxidative stress and proinflammatory marker in metabolic syndrome using a highfructose diet induced rat model. Biomed Pharmacol J 2019; 12:305-24. doi:10.13005/bpj/1642

[29] Koroglu KM, Cevik O, Sener G, Ercan F. Apocynin alleviates cisplatin-induced testicular cytotoxicity by regulating oxidative stress and apoptosis in rats. Andrologia. 2019; 51:e13227. doi: 10.1111/and.13227

[30] Johnsen SG. Testicular Biopsy Score Count - A Method for registration of spermatogenesis in human testes: normal values and results in 335 hypogonadal males. Hormones 1970; 1:2-25. doi: 10.1159/000178170

[31] Chen SJ, Allam JP, Duan YG, Haidl G. Influence of reactive oxygen species on human sperm functions and fertilizing capacity including therapeutical approaches. Arch Gynecol Obstet 2013; 288:191-9. doi: 10.1007/s00404.013.2801-4 